\title{
Therapeutic angioembolization of bleeding pancreaticoduodenal artery pseudoaneurysm following laparoscopy assisted Frey’s procedure
}

\author{
J Saravanan, R Sukumar, S Jeswanth, P Ravichandran
}

\begin{abstract}
Introduction: Minimal access surgery has revolutionized surgery and is considered the gold standard of management for most surgical pathologies of the abdomen. We present a case which to our best knowledge is the first ever report of the non-surgical management of a pancreaticoduodenal artery (PDA) pseudoaneurysm following laparoscopy assisted Frey's procedure (LAFP). Case Report: A 38-year-old male patient was transferred to our hospital due to persistent gastrointestinal bleeding following LAFP. He underwent LAFP for chronic calcific pancreatitis (CCP) two weeks prior to his transfer. Computed tomography angiogram showed the source of bleeding to be a branch of superior pancreaticoduodenal artery (SPDA). The patient was taken to interventional radiology where successful superselective microcoil embolization was performed. We present this rare case of pancreaticoduodenal pseudoaneurysm along with a review of medical
\end{abstract}

J Saravanan 1, R Sukumar², S Jeswanth ${ }^{1}$, P Ravichandran ${ }^{1}$ Affiliations: ${ }^{1}$ Institute of Surgical Gastroenterology \& Liver Transplantation, Centre for GI Bleed, Division of HPB diseases, Stanley Medical College Hospital, Old Jail Road, Chennai, India; 'Department of Interventional Radiology, Institute of Surgical Gastroenterology \& Liver Transplantation, Centre for GI Bleed, Division of HPB diseases, Stanley Medical College Hospital, Old Jail Road, Chennai, India.

Corresponding Author: Dr. Saravanan J, Institute of Surgical Gastroenterology \& Liver Transplantation, Centre for GI Bleed, Division of HPB diseases, Stanley Medical College Hospital, Old Jail Road, Chennai, India; Email: saran_world@yahoo.com

Received: 09 October 2012

Accepted: 17 November 2012

Published: 01 May 2013 literature. Conclusion: A prudent selection of the operative candidate for laparoscopic pancreatic surgery and a high degree of vigilance is imperative to circumvent avoidable morbidity and mortality.

Keywords: Laparoscopy, Frey's procedure, Complications, Pancreaticoduodenal artery, Gastrointestinal bleeding, Angiography, Pseudoaneurysm

$$
* * * * * * * * *
$$

Saravanan J, Sukumar R, Jeswanth S, Ravichandran P. Therapeutic angioembolization of bleeding pancreaticoduodenal artery pseudoaneurysm following laparoscopy assisted Frey's procedure. International Journal of Case Reports and Images 2013;4(5):275-278.

$$
* * * * * * * * *
$$

doi:10.5348/ijcri-2013-05-311-CR-8

\section{INTRODUCTION}

Gastrointestinal bleeding due to pseudoaneurysm is a rare but potentially fatal complication of surgery. Early recognition and management of this complication is critical [1]. Although surgery has traditionally been the treatment of choice, therapeutic angiography is emerging as a safe and effective treatment modality [1, 2]. In this article we present a case, where we managed a pseudoaneurysm of the pancreaticoduodenal artery following laparoscopy assisted Frey's procedure (LAFP). This to our knowledge is the first such case report in English literature. We discuss the pathophysiology of the condition, the treatment options and our rationale for opting for angiographic intervention. 


\section{CASE REPORT}

A 38-year-old male was brought to the emergency department with acute upper gastrointestinal bleeding. two years prior to this admission he was diagnosed with chronic calcific pancreatitis (CCP). The CCP was being managed symptomatically with gradual improvement of the symptoms. One month ago he was admitted to a local hospital with intense abdominal pain. He was evaluated and subjected to a LAFP. On the fourteenth postoperative day he had multiple bouts of massive hematemesis along with malena. He was transfused multiple units of packed blood cells and was transferred to our institution. On arrival at the emergency department, the patient was in hypovolemic shock with a heart rate of 120 beats per minute and a blood pressure of 9o/60 mmHg. His hemoglobin level was $7 \mathrm{~g} / \mathrm{dL}$. A nasogastric tube was inserted which drained frank blood. He was aggressively resuscitated and treated with intravenous pantoprazole and multiple units of packed cells. After the patient was stabilized, computed tomographic angiography was done which demonstrated a giant pseudoaneurysm of one of the branches of superior pancreaticoduodenal artery. He was taken to the angiography suite and through a right transfemoral approach the celiac trunk and the superior mesenteric artery were cannulated. The SPDA was cannulated with a super selective micro-catheter. Angiogram revealed a pseudoaneurysm measuring $3 \times 2.4 \mathrm{~cm}$ arising from a branch of SPDA with extravasation of contrast into duodenal lumen (Figures $1,2)$. Multiple 0.018 inch microcoils were deployed into the feeding vessel and also into the SPDA proximal and distal to the feeding vessel (Figure 3). Post embolization check angiogram revealed free flow of contrast into the

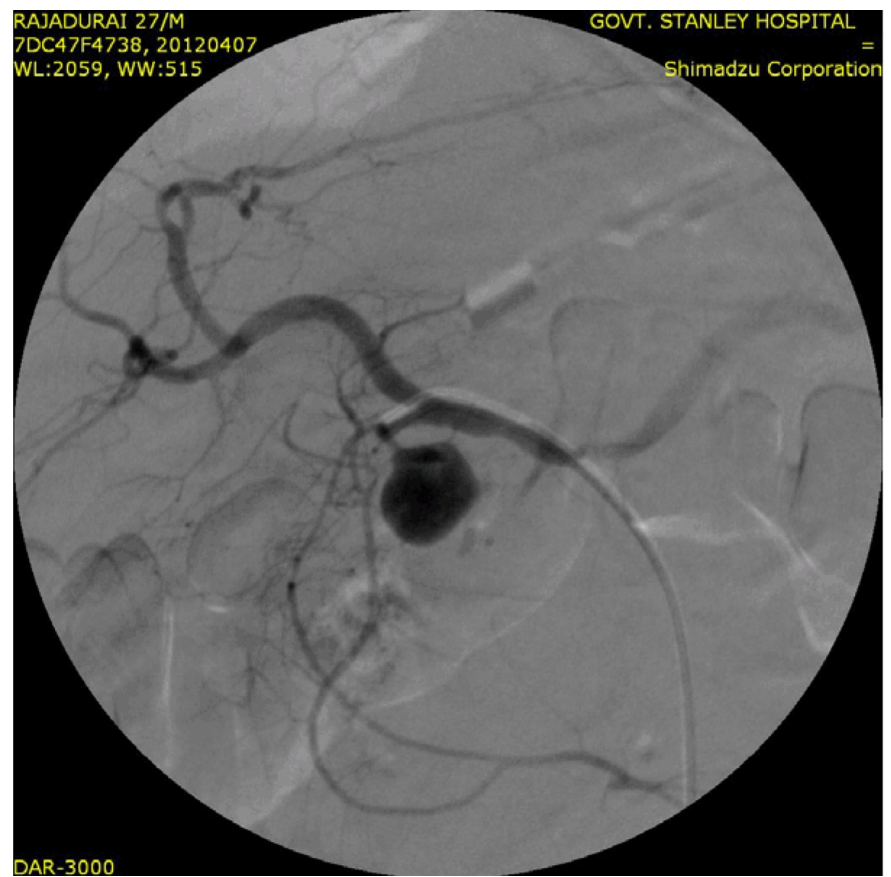

Figure 1: Pre-embolization gastroduodenal artery injection into pseudoaneurysm visualized in superior pancreaticoduodenal artery.

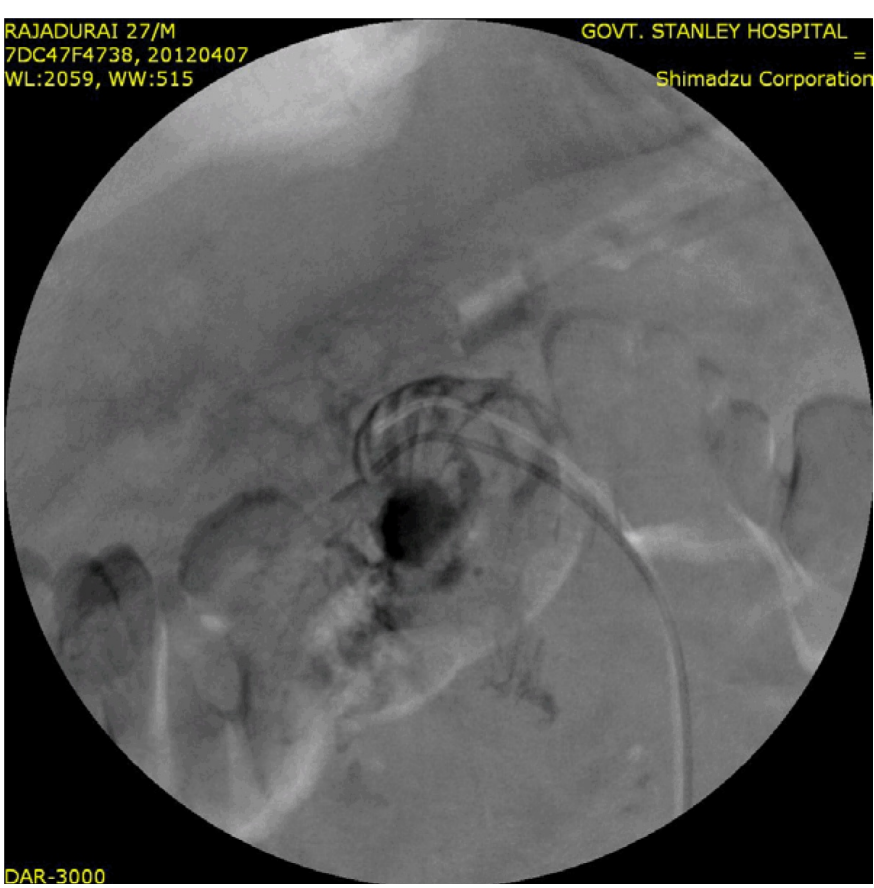

Figure 2: Contrast extravasation from pseudoaneurysm into C loop of duodenum.

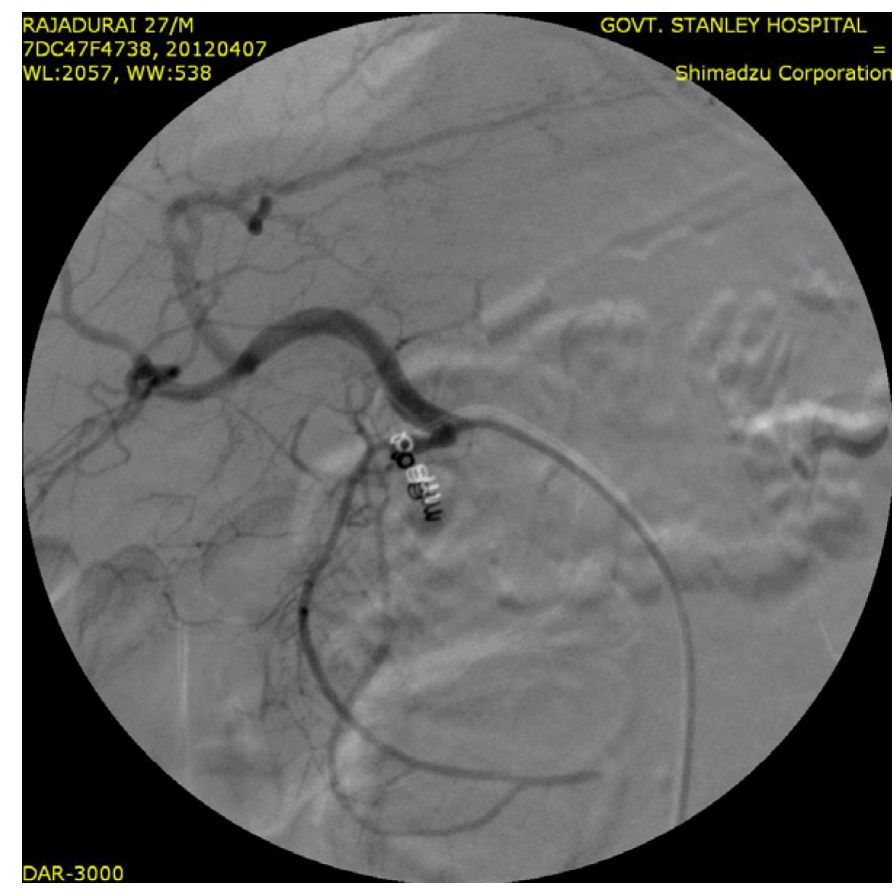

Figure 3: Microcoils visualized in post-embolization check angiogram.

gastroduodenal artery (GDA), hepatic artery, branches of the superior mesenteric artery. The procedure was uneventful without any complications. The postprocedure follow-up color Doppler ultrasound showed no flow within the lumen of the pseudoaneurysm with collapse of pseudoaneurysmal cavity. He was discharged on the 14th day of hospitalization. On latest follow-up, the patient was asymptomatic. 


\section{DISCUSSION}

Pseudoaneuryms of visceral arteries are uncommon (5-10\%) but occur as critical complications following pancreatitis and pancreatic surgery $[1,2]$. Early diagnosis and adequate therapeutic interventions are imperative in these cases. A giant pseudoaneurysm is defined as a pseudoaneurysm equal to or greater than 5 $\mathrm{cm}$ in size $[1,3]$. The visceral arteries which most commonly develop pseudoaneurysm are the splenic artery, hepatic arteries, gastric and gastroepiploic arteries, gastro-duodenal artery and branches of the mesenteric arteries $[1,2,4]$. Activation and release of pancreatic enzymes causes the rupture of membrana elastica interna of the splanchnic vessels followed by segmental thrombosis of the vessel $[1,3-5]$. Thrombosis of the vasa vasorum causes ischemia of the arterial wall with resultant necrosis. Massive bleeding occurs if the weakened vessel wall suddenly ruptures $[1$, 3-5]. A pseudoaneurysm develops when the rupture is contained and the hematoma is surrounded by a reactive fibrous capsule. Gastroduodenal artery aneurysms are extremely rare, comprising only $1.5 \%$ of all reported peripheral aneurysms, with pancreaticoduodenal artery pseudoaneurysms being much rarer $[1$, $2,6-8]$.

Contrast enhanced computed tomography provides optimal visualization of these vascular abnormalities [3]. The demonstration of a homogeneously enhancing structure within or adjacent to a pseudocyst or contiguous with a vascular structure in a case of pancreatitis is highly suggestive of an associated pseudoaneurysm [3]. Angiography serves an important role for both diagnostic and therapeutic purposes as shown in our case [5, 7, 8]. Early diagnosis of pseudoaneurysms is important because of the risk of rupture and subsequent mortality. There are multiple etiologies for the development of pseudoaneurysms including atherosclerosis, trauma, surgery, pancreatitis, infection, collagen vascular disease and congenital abnormalities $[1,2,4,6]$. Patients may be asymptomatic or present with symptoms of gastrointestinal bleeding, intraperitoneal hemorrhage, obstructive jaundice due to external pressure by the pseudoaneurysm or hemobilia $[1,2,6,7]$.

A review of visceral aneurysms conducted by Moore et al. concluded that $35 \%$ of GDA aneurysms are ruptured at presentation, carrying a $21 \%$ mortality rate [6]. In the past, these pseudoaneurysms were treated surgically $[2,6]$. However, in the last two decades, radiographic intervention has emerged as an attractive method for treating these patients. Surgical treatments include resection of the aneurysm and placing an interposition graft or performing aneurysmectomy with or without patching using a great saphenous vein $[2,6]$. Endovascular treatments used are embolization or stent graft repair of the aneurysm $[5,7,8]$. The potential complications of angiography, aside from those of standard femoral cannulation include infarction of viscera and abscess formation. Fortunately, the infarction rate is low due to the rich collateral blood supply [6]. Saltzberg et al. reported an initial technical success rate of $94.4 \%$ for the endovascular procedures. [7]. The angiographic principle of occluding the artery upstream and downstream (front and backdoor concept) from the origin of the lesion should be adopted [5-8]. Therefore, it is necessary to perform embolization proximally and distally to the pseudoaneurysm, as well as within the pseudoaneurysm itself, if required, to exclude it from both arterial backflow and antegrade flow. Bleeding into the peritoneal space or the gastrointestinal tract beginning several days following an operation should be a cause for suspicion for the possibility of a ruptured pseudoaneurysm [1, 4, 8, 9]. Management of pseudoaneurysms may be more non-selective as compared to that of a classical aneurysm; as the length or size of the neck of the pseudoaneurysm does not alter the technique of tackling it. Due to the artifacts produced by coils on CECT, duplex ultrasound has been recommended for follow-up in these post embolization patients $[2,4,6]$.

Our patient developed the pseudoaneurysm due to a leak occurring after LAFP which initiated an infectious/inflammatory process in the region of the GDA causing damage to the vessel wall. Any operation which involves handling of an infected space or organ is a risk factor for infecting the peritoneal cavity and development of a pseudoaneurysm [1, 2, 4]. Thus, vessel anatomy, location, etiology of the disease and the patient's underlying condition must be taken into account in the decision to embark on endovascular or surgical therapy $\left[1,2,5^{-7}\right]$. A surgical ligation would be more appropriate in patients who are unsuitable or unstable for emobolizations in our patient [2, 6, 7]. In cases of intraperitoneal rupture with hypotension, open surgery is the management of choice [2, 6, 7]. Angioembolic occlusion of the aneurysm provided an effective treatment with minimal morbidity risk. Surgical repair in our case would have been particularly challenging and possibly morbid as it would have required re-exploring a previously operated space.

\section{CONCLUSION}

Pancreaticoduodenal artery pseudoaneurysms are a very rare complication of pancreatic surgery and carry a very high risk of mortality. A very high index of suspicion is required to identify this potentially disastrous complication postoperatively. Non surgical management in the form of angiographic embolization is not only a feasible but possibly the preferred treatment modality in cases of visceral artery pseudoaneurysms.

$$
* * * * * * * * *
$$

\section{Author Contributions}

J Saravanan - Substantial contributions to conception and design, Acquisition of data, Analysis and interpretation of data, Drafting the article, Revising it 
critically for important intellectual content, Final approval of the version to be published

$\mathrm{R}$ Sukumar - Analysis and interpretation of data, Drafting the article, Revising it critically for important intellectual content, Final approval of the version to be published

S Jeswanth - Analysis and interpretation of data, Drafting the article, Revising it critically for important intellectual content, Final approval of the version to be published

P Ravichandran - Analysis and interpretation of data, Drafting the article, Revising it critically for important intellectual content, Final approval of the version to be published

\section{Guarantor}

The corresponding author is the guarantor of submission.

\section{Conflict of Interest}

Authors declare no conflict of interest.

\section{Copyright}

(C) J Saravanan et al. 2013; This article is distributed under the terms of Creative Commons Attribution 3.0 License which permits unrestricted use, distribution and reproduction in any means provided the original authors and original publisher are properly credited. (Please see www.ijcasereportsandimages.com /copyright-policy.php for more information.)

\section{REFERENCES}

1. Sato N, Yamaguchi K, Shimizu S, et al. Coil embolization of bleeding visceral pseudoaneurysms following pancreatectomy: the importance of early angiography. Arch Surg 1998;133(10):1099-102.

2. Pulli R, Dorigo W, Troisi N, Pratesi G, Innocenti AA, Pratesi C. Surgical treatment of visceral artery aneurysms: A 25-year experience. J Vasc Surg 2008;48(2):334-42.

3. Balthazar EJ, Fisher LA. Hemorrhagic complications of pancreatitis: radiologic evaluation with emphasis on CT imaging. Pancreatology 2001;1(4):306-13.

4. Stabile BE, Wilson SE, Debas HT. Reduced mortality from bleeding pseudocysts and pseudoaneurysms caused by pancreatitis. Arch Surg 1983;118(1):45-51.

5. Salam TA, Lumsden AB, Martin LG, Smith RB 3rd. Nonoperative management of visceral aneurysms and pseudoaneurysms. Am J Surg 1992;164(3):215-9.

6. Moore E, Matthews MR, Minion DJ, et al. Surgical management of peripancreatic arterial aneurysms. J Vasc Surg 2004;40(2):247-53.

7. Saltzberg SS, Maldonado TS, Lamparello PJ, et al. Is endovascular therapy the preferred treatment for all visceral artery aneurysms? Ann Vasc Surg 2005;19(4):507-15.

8. Chong WW, Tan SG, Htoo MM. Endovascular treatment of gastroduodenal artery aneurysm. Asian Cardiovasc Thorac Ann 2008;16(1):68-72.

9. Nonokuma M, Okazaki M, Higashibara $\mathrm{H}$, et al. Successful embolization of pancreaticoduodenal artery pseudoaneurysm in a patient with common hepatic arterial occlusion after modified pancreatoduodenectomy with preservation of arteries in the head of pancreas. Hepatogastroenterology 2009;56(89):245-8.
Access full text article on other devices

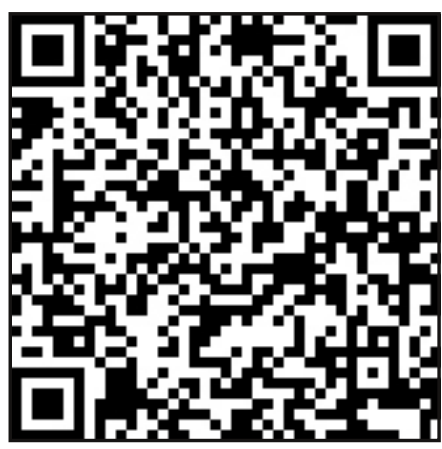

Access PDF of article on other devices

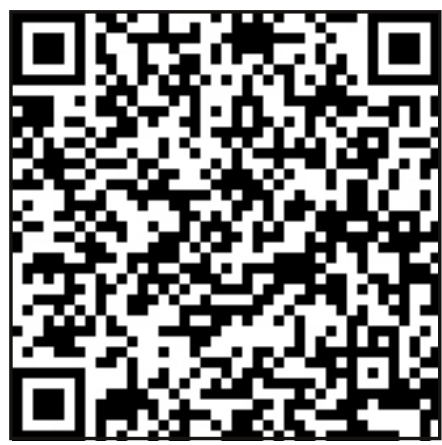

